\title{
Intérêt de l'indométacine comme traitement d'épreuve des céphalées hémi-faciales : aspects cliniques et pharmacologiques
}

\author{
A. Sulukdjian (Courbevoie), D. Nguyen (Paris), V. Luciani (Montrouge), A. Chanlon (Paris), \\ N. Moreau (Paris)
}

Arek SULUKDJIAN ${ }^{1}$, Diane NGUYEN², Vanina LUCIANI ${ }^{3}$, Audrey CHANLON², Nathan MOREAU ${ }^{4,5}$

1- Etudiant en $5^{\text {ème }}$ année de chirurgie dentaire, Faculté de Chirurgie dentaire, Université Paris Descartes

2- Praticien attaché, consultation de diagnostic et traitement des douleurs chroniques oro- faciales, service de médecine bucco-dentaire,HôpitalBretonneau,AP-HP,Paris

3- Etudiante en 6ème année de chirurgie dentaire, Faculté de Chirurgie dentaire, Université Paris Descartes

4- Responsable de la consultation de diagnostic et traitement des douleurs chroniques oro-faciales, service de médecine bucco- dentaire, Hôpital Bretonneau, AP-HP, Paris

5- MCU-PH en médecine et chirurgie orale, Faculté de Chirurgie Dentaire, Université Paris Descartes \& Service de Médecine buccodentaire, Hôpital Bretonneau, AP-HP, Paris \& Laboratoire de Neurobiologie Oro-Faciale, Université Paris Diderot

La région oro- faciale peut être le siège de nombreuses pathologies douloureuses dont certaines d'origine neurovasculaire (migraine) ou trigémino-autonomiques (algie vasculaire de la face, hémicrânie paroxystique) peuvent être de diagnostic difficile, surtout en cas de présentation purement faciale. L'hémicrânie paroxystique (HP) est une céphalée primaire caractérisé e par de multiples crises douloureuses unilaté rales associé es à des troubles autonomiques crânio-faciaux (ICHD3). De prédominance féminine, sa prévalence est estimée à $1 / 50000$. Son âge moyen d'apparition est en moyenne à 40 ans et touche une population de 5 à 68 ans. La grande particularité de cette pathologie est que son traitement de référence est aussi son principal outil de confirmation diagnostique. En effet, l'HP présente une réponse complète à l'indométacine, ce qui pourrait permettre ainsi d'écarter les autres pathologies douloureuses faciales. Cet AINS a pour particularité d'être le seul à avoir une action spécifique au niveau hypothalamique, dont l'activation serait responsable du tableau céphalalgique dans l'hémicrânie paroxystique. A ce titre, il peut s'avérer un test diagnostique intéressant en cas de douleurs hémifaciales inexpliquées, en particulier si des signes autonomiques sont présents. Il est rapporté trois cas de patients ayant consulté dans la consultation douleurs chroniques oro- faciales du service de médecine buccodentaire de l'hôpital Bretonneau pour des douleurs oro-faciales inexpliquées associées à de discrets signes dysautonomiques (sudation, obstruction nasale, rhinorhée, larmoiement). Alors que leurs précédents traitements étaient peu ou pas du tout efficaces (antalgiques de pallier 2, carbamazépine ou gabapentine), un traitement d'épreuve par indométacine a permis une disparition immédiate de leurs douleurs faciales. La présentation clinique et la réponse absolue à l'indométacine ont ainsi permis de poser le diagnostic d'hémicrânie paroxystique chez ces trois patients. Les contre-indications de la molécule étant réduites à celles de sa famille pharmacologique, cette série de cas suggère l'intérêt de l'utilisation l'indométacine comme test diagnostique chez tout patient souffrant de céphalées hémifaciales strictes, surtout en présence de signes autonomiques.

Headache Classification Commitee of the IHS. The International Classification of Headache Disorders, 3rd edition (beta version). Cephalalgia 2013;33(9):629-808. 